\title{
The Wise Know: Decline Tender Loving Care and Respect Silver Hair
}

\section{Petros Levounis}

Received: 22 October 2014 / Accepted: 6 November 2014 /Published online: 9 December 2014

(C) Academic Psychiatry 2014

To the Editor:

The Diagnostic and Statistical Manual of Mental Disorders, 5th Edition (DSM-5) [1], establishes 11 criteria for the diagnosis of substance use disorders. Although the criteria are very well researched and widely used in clinical practice, they are not easy to memorize. This short communication proposes a whimsical mnemonic to help students and practitioners commit the 11 DSM-5 criteria to memory.

The 11 criteria may be summarized and grouped in four categories:

1. Physiology:

Tolerance

Withdrawal

2. The core problem of substance use disorders:

Knowledge of adverse consequences, yet continued use

3. Internal preoccupation:

Desire to cut down

Time — a great deal of time — spent using
Larger amounts or longer periods of use than intended

Craving

4. External consequences:

Activities given up

Role obligations neglected

Social or interpersonal problems

Hazardous use

The acronym TW - K - DTLC - ARSH lends itself to the somewhat silly and largely nonsensical (but hopefully memorable and not unpleasant) phrase "The Wise Know: Decline Tender Loving Care and Respect Silver Hair."

\section{Reference}

1. American Psychiatric Association: Diagnostic and Statistical Manual of Mental Disorders, 5th Edition. Arlington, VA, American Psychiatric Association, 2013

P. Levounis $(\bowtie)$

Rutgers New Jersey Medical School, Newark, NJ, USA

e-mail: PetrosLevounis@msn.com 